\title{
Flavin Adenine Dinucleotide (FAD) Pegylated (PEG)- Complexes: Proof of Concept (PoC) of theranostic tool on a Murine Breast Cancer Model
}

\author{
Celia Arib ${ }^{1^{*}}$, Hui Liu ${ }^{2 *}$, Qiqian Liu ${ }^{1}$, Anne-Marie Cieutat ${ }^{1}$, Didier Paleni ${ }^{3}$, Xiaowu Li ${ }^{2 凶}$, Jolanda Spadavecchia ${ }^{1,2 \bowtie}$ \\ 1. CNRS, UMR 7244, NBD-CSPBAT, Laboratoire de Chimie, Structures et Propriétés de Biomatériaux et d'Agents Thérapeutiques Université Sorbonne Paris \\ Nord, campus Bobigny, France. \\ 2. Department of Hepatobiliary Surgery, Guangdong Provincial Key Laboratory of Regional Immunity and Diseases \& Carson International Cancer Center, \\ Shenzhen University General Hospital \& Shenzhen University Clinical Medical Academy Center, Shenzhen University, Shenzhen, China. \\ 3. BioEVEN start-up, 75 rue de Lourmel 75015 Paris, France. \\ *Authors contributed equally to this work. \\ \Corresponding authors: E-mail: lixw1966@163.com; jolanda.spadavecchia@univ-paris13.fr.
}

() The author(s). This is an open access article distributed under the terms of the Creative Commons Attribution License (https://creativecommons.org/licenses/by/4.0/). See http://ivyspring.com/terms for full terms and conditions.

Received: 2021.06.03; Accepted: 2021.08.08; Published: 2022.01.01

\begin{abstract}
Flavin adenine dinucleotide (FAD) plays a key role in an extensive range of cellular oxidation-reduction reactions, which is engaged in metabolic pathways. The purpose of this study was to realize pegylated flavins formulation, named FAD and FAD-PEG diacid complex as theranostic tool in cancer therapy. For this objective, a murine breast cancer model, which was induced by mouse-derived4T1 breast cancer cells was studied to assess the therapeutic efficacy of FAD (named NPI) and FAD-PEG diacid complex (named NP2). The cytokines were monitored to evaluate the serum inflammatory factors to develop the blood cell content of different groups of nude mice. The experimental model shows that an intravenous injection of FAD (NPI) can significantly reduce tumour volume, tumour index and thymus index, and decrease neutrophils (NE), monocytes (MO), eosinophils (EO), and basophils (BA). At the same time, the content of IL-1 $\alpha$, IL-12P70, TNF $\alpha$, IL-1 $\beta$ and IL- 6 was significantly reduced, and the content of IL- 10 was significantly increased. These results provide the proof-of-concept for FAD as a smart adjuvant for cancer therapy and encourages their further development in the field of Nanomedicine.
\end{abstract}

\section{Introduction}

Breast cancer is the most commonly occurring cancer in women and overall the second most common cancer in the world $[1,2]$. There are different limitations to conventional therapy for breast cancer [3]. Nanocarriers have the potential to provide a high drug loading capacity, stability, exceptional tolerability, drug deterioration, decrease multidrug resistance, controlled release and encouraged delivery of anticancer drugs [4]. Consequently, therapeutics and theranostics drug delivery nanocarrier systems have significant benefits over common treatment methods [5]. Nevertheless, there are many challenges needed to be resolved in drug delivery systems, such as undue accumulation of the carriers in the liver, poor therapeutic efficacy on the cancer cells and the barriers near the tumour areas or in the vasculature area; all of these were realized as the obstacles to penetrate into cancer cells and are needed to be resolved. Flavin adenine dinucleotide (FAD) plays a part in various metabolic reactions where the biological function is naturally connected to its structure conformation change [6]. Its main role is to be a cofactor, which is required for the activity of several flavoproteins, and is important for electron transport pathways in many living systems $[7,8]$. FAD synthase is the last enzyme in the metabolic pathway producing $\mathrm{FAD}$, a protein confined in both cytosol and in mitochondria $[9,10]$ and it regulates different 
processes for cell life and death, resulting in ROS production, antioxidant defence, protein folding, and chromatin remodelling [10]. Recent studies show that the modifications in coenzyme levels have been recorded in several cancers [11]. Coenzymes take part in regulating enzyme activity to perform disparate biochemical reactions [12,13]. Mutations in metabolic enzymes obstruct, typically, biochemical reactions leading to many disorders [14-16]. Recently J. Spadavecchia and D. Paleni, conceived for the first time, the role of FAD cofactor as nanodevice in cancer therapy [17]. The first aim of this present study, was the evolution of novel adjuvant in chemotherapy by using FAD alone or complexed to pegylated chains to obtain hybrid complex (FAD PEG-Diacid) and compared with a common chemotherapeutic (5 Fluro Uracil; 5-FU) in the therapy of cancer. For this purpose, a mouse breast cancer model induced by mouse-derived $4 \mathrm{~T} 1$ breast cancer cells, was used to evaluate the therapeutic efficacy of FAD (named NP1) and FAD-PEG diacid complex (named NP2). We believe that this study is important for planning optimal chemotherapeutic systems in order to comprehend the mechanisms of poor effects of 5-FU.

\section{Materials and Methods}

\section{Methods}

To evaluate the therapeutic effects of FAD (NP1) and FAD/PEG complex (NP2) in the 4T1 cells induced breast cancer model of mouse. Animals were divided into a sham group, $0.9 \% \mathrm{NaCl}$ injection group, PEG group, NP1 solution intravenous injection group, NP2 solution intravenous injection group, NP1 solution oral administration group, NP2 solution oral administration group, and positive control 5 -fluorouracil group, a total of 8 groups. An injection of $4 \mathrm{~T} 1$ cells containing matrigel was administered into the second pair of breast pads on the left side of the mouse (the total number of injected cells is $2 \times 10^{6}$ ). When the tumour model is successfully built, $100 \mu \mathrm{L}$ of the drug is administered intravenously and orally, twice a day (Fixed at 9:00 am and 3:00 pm every day, 6 hours apart), 2 days a week (once every 3 days), for 3 weeks. The blood was collected after the animals were anesthetized by ketamine/xylazine. The tumours were removed, weighed, and the percentage of inhibition of the drug was calculated with a negative control. The liver, kidney, spleen, and thymus were dissected to calculate the ratio (index) to weight. Furthermore, whole blood count was detected using a haemocytometer before 1 week of $4 \mathrm{~T} 1$ cell injection and before the first drug administration (DIV 8), 1 week (DIV 15), 2 weeks (DIV 22) and 3 weeks (DIV 29) number of cells. Flow cytometry was used to measure serum cytokines before $4 \mathrm{~T} 1$ cells (DIV 0), 1 weeks after $4 \mathrm{~T} 1$ cells were injected (DIV 8), 2 weeks after DIV15, 3 weeks (DIV 22), and 4 weeks (DIV 29).

\section{Materials}

The Flavin Adenine Nucleotide, Dicarboxylic Poly Ethylene Glycol (PEG)-600, cell culture media, fetal bovine serum (FBS), and penicillin-streptomycin were obtained from Thermo Fisher Scientific (Waltham, MA, USA). The 5-fluorouracil (5-FU) was purchased from Shanghai Xudong Haipu Pharmaceutical Co., Ltd. (Shanghai, People's Republic of China).

The Bio Legend LEGENDplex ${ }^{\mathrm{TM}}$ multiplex bead-based assay kit was from BioLegend (San Diego, CA, USA). The Matrige ${ }^{\circledR}$ Basement Membrane Matrix was from Corning (Tewksbury, MA, USA). HEMAVET950FS animal blood analyzer special reagents were from Drew Scientific, Inc., USA. Isoflurane was obtained from RWD Life Science Co., Ltd. (Shenzhen, People's Republic of China).All other reagents were either obtained from Sigma-Aldrich (St. Louis, MO, USA) or noted otherwise.

\section{Instruments}

Animal Weight Balance (Mettler - Toledo instruments (Shanghai) Co., LTD, Serial number: PL3001-s), Drug Weighing Balance Serial number: AL204 Electronic Balance Mettler-Toledo instruments (Shanghai) Co., LTD. HEMAVET 950 Animal Blood Analyzer (Drew Scientific, Inc., USA- Model: HEMAVET 950FS), Automatic biochemical analyzer (Hitachi-Model: 7100), BD Accuri C6 Flowcytometer (BD company), High Resolution Small Animal Ultrasound Imaging System (Visual Sonics-Model: Vevo2100, probe MS400, frequency $30 \mathrm{MHz}$ ), Inductively Coupled Plasma Mass Spectrometer (ICAP-Q) (American Thermo Company).DB-3EFS type hot plate (Tianjin Gongxing Laboratory Instrument Co., Ltd), Milli-Q ultrapure water treatment system (American Millipore Company).

\section{Synthesis Procedures of FAD-PEG Diacid Complex (NP2)}

The synthesis of FAD-PEG Diacid Complex (NP2) was described previously [18].

\section{Physical-Chemical characterization}

All characterizations were carried out in triplicate determinations as described previously [4-18].

\section{Mice Tests}

In vivo tests were performed using male nude mice BALB/c mice, Grade: SPF, age 5 weeks. Animals were randomly divided into eight groups: the sham 
group ( $\mathrm{n}=10)$, the $0.9 \% \mathrm{NaCl}$ injection group $(\mathrm{n}=8)$, the PEG group $(n=8)$, the NP1 solution intravenous group $(n=8)$, the NP2 solution intravenous group $(n=8)$, the NP1 solution oral administration group $(n=8)$, the NP2 solution oral administration group $(n=8)$ and the 5 -fluorouracil group as positive control $(n=7)$. Animal production license number: SCXK (Yue) 2018-0034, Guangdong Medical Laboratory Animal Center; animal certificate number: No. 44005800009527; No. 44005800009345; animal Use License number: SYXK (Yue) 2018-0001, Laboratory animal Center, Guangzhou University of Chinese Medicine. The technical procedure was described previously [18].

\section{Quarantine}

All experiments and technical procedures were recorded and described as in previous paper [18].

\section{Grouping and Administration}

After the tumour model of nude mice was built. $100 \mu \mathrm{L}$ of different test drugs were intravenously and orally administered twice every day (injection at 9:00 am and at 15:00 pm). The drug was administered two days every week (dosing every three days) and for a total of 3 weeks.

Table 1. Experimental animals were randomly divided into 8 groups: Experimental conditions of FAD formulations (NP1; NP2) administration

\begin{tabular}{lllll}
\hline Group & Dose drugs & $\begin{array}{l}\text { Number of } \\
\text { mice }\end{array}$ & $\begin{array}{l}\text { Method of } \\
\text { administration }\end{array}$ & $\begin{array}{l}\text { Administration } \\
\text { frequency }\end{array}$ \\
\hline Sham & Saline & 10 & i.v. & B.i.d \\
Saline & Saline & 8 & i.v. & B.i.d \\
PEG & PEG & 8 & i.v. & B.i.d \\
NP 1-i.v. & $10 \mathrm{mg} / \mathrm{kg}$ & 8 & i.v. & B.i.d \\
NP 2-i.v. & $10 \mathrm{mg} / \mathrm{kg}$ & 8 & i.v. & B.i.d \\
NP 1-i.g. & $10 \mathrm{mg} / \mathrm{kg}$ & 8 & i.g. & B.i.d \\
NP 2-i.g. & $10 \mathrm{mg} / \mathrm{kg}$ & 8 & i.g. & B.i.d \\
5-FU & $10 \mathrm{mg} / \mathrm{kg}$ & 7 & i.v. & Q.d \\
\hline
\end{tabular}

\section{Detection Indicators}

Observation of the general condition, body weight and organ index of nude mice. The general situation of the nude mouse includes the activity, mental state, skin color, diet, water intake and urine output. Body weight (BW) was weighed every 3 days. At the end of the experiment, the heart, liver, spleen, lung, kidney and tumour were separated and accurately weighed after the nude mice were sacrificed, and the heart, liver, spleen, lung, kidney and tumour index were calculated.

That is:

Tissue weight $(m g) /$ body weight $(g)=$ Tissue weight/BW.

\section{Organ index detection}

Body weight (BW) was weighed every 3 days. At the end of the experiment, the heart, liver, spleen, lung, kidney and tumor were separated and accurately weighted after the nude mice were sacrificed. The heart, liver, spleen, lung, kidney and tumor index were calculated by using the following formula:

$$
\text { Organ index }(\mathrm{mg} / \mathrm{g})=\text { Tissue weight } / \mathrm{BW} \text {. }
$$

\section{Tumor volume assessment}

The growth of tumors was monitored every 3 days by caliper measurement of tumor length (a) and width (b). Tumor volume was estimated by using the following formula:

$$
\text { Tumor volume }\left(m m^{3}\right)=\left(a^{*} b^{2}\right) / 2 \text {. }
$$

The Table of results was reported in Supporting Information (Table S1).

\section{Whole blood cell count detection}

Whole blood was measured as previously described [18].

\section{Multiplex bead-based assay for pro-inflammatory cytokine}

Flow cytometry was used to detect serum cytokine levels as previously described [18].

\section{Sample preparation}

$250 \mu \mathrm{L}$ of assay buffer was added to the standard to form a liquor of $10000 \mathrm{pg} / \mathrm{mL}$, and left at room temperature for 10 minutes; 8 tubes were taken, numbered C1-C7, $75 \mu \mathrm{L}$ of assay buffer added to each tube, and then $25 \mu \mathrm{L}$ of standard stock solution was added to $\mathrm{C} 1$ tube. After vortexing, $25 \mu \mathrm{L}$ was transferred to $\mathrm{C} 2$ tube. By analogy to $\mathrm{C} 6,75 \mu \mathrm{L}$ was retained in each tube.

\section{Flow cytometry}

Flow cytometry analysis was carried out as previously described [18].

\section{Statistical analysis}

Statistical analysis was performed by GraphPad Prism 8.0 software as previously described [18].

\section{Results and Discussion}

Recently we have proved the ability of FAD formulations (NP1; NP2) as adjuvant in chemotherapy against hepatocarcinome [18]. In particular we blended NP1-NP2 with 5-FU in which electrostatic charges between the chemical groups of the drug and FAD formulations accomplished the final hybrid formulation. On the basis of these hypothesis, we believe that NP1-NP2 under complex form, after internalization in cancer cells, could improve the therapeutic efficacy, due to a double action on nuclei 
and mitochondria for blocking the binding of aminoacyl-tRNA to the mRNA-ribosome complex with evident variation in driven force and electrolytic condition. It has been suggested that the expression of Glucose 6 phosphate dehydrogenases (G6PD) and transketolase (TKT) are positively correlated to the decreased overall and relapse-free survival in breast cancer. The synergic action of FAD-5FU formulation inhibits Glucose 6 phosphate dehydrogenases $[19,20]$ and dihydropyrimidine (DPD) [21] with a simultaneous reparation, and inhibition of resistance and improvement of anticancer activity. For this purpose, we conceived a lot of experiments in which NP2 was tested as pegylated complex and compared with control (FAD; i.e. NP1) and anti-cancer drug (5-Fluoro-Uracile [5-FU]).

\section{FAD (NP1) and FAD/PEG complexes (NP2) did not affect body weight changes in mice with breast cancer}

Breast cancer mice induced by $4 \mathrm{~T} 1$ cells were used as a research model to evaluate the therapeutic effects of FAD(NP1) and FAD/PEG (NP2) complexes. The mice were weighed every 3 days. As shown in Fig. 1, the mice injected with 4T1 cells showed a different degree of decline compared with the sham group, the saline and PEG groups, the different administration methods of FAD (NP1) and FAD/PEG (NP2) complexes did not affect body weight of breast cancer model mice. However, the positive control drug 5-FU group, showed significant weight loss after 1 week of prophylactic administration, and had a statistical significance in DIV 18, DIV 21, DIV 24, DIV 27 and DIV $30(\mathrm{p}<0.05)$.

\section{FAD (NP1) intravenous injection significantly reduces tumour volume in mice}

The tumour length and width of mice were measured every 3 days. As shown in Fig. 2, the volume of the $4 \mathrm{~T} 1$ cell injection group gradually increased, and the NP1 and NP2 intravenous injection groups could delay the tumour volume increase. At the end of the experiment, compared with the first tumour volume, the tumour volume increased by 5.34 times, and the PEG group increased by 5.37 times. However, the tumour volume of the NP1 intravenous group and the NP2 intravenous group increased by 4.07 times and 4.50 times, respectively, which was significantly statistically different from the saline group and the PEG group $(p<0.001)$. The tumour volume of mice in the 5-FU group was significantly smaller than that in the saline group and the PEG group, which had significant statistical significance $(\mathrm{p}<0.001)$.

\section{FAD intravenous injection significantly reduces tumour and spleen size in mice}

At the end of the experiment, mouse tumours and spleen tissues were isolated and photographed. As shown in Figure S1 in Supporting Information, the tumour volume of mice in the NP1 and NP2 intravenous injection groups were smaller than that in the saline and PEG groups.

\section{FAD intravenous injection significantly reduces tumour index and spleen index}

At the end of the experiment, the mice in each group were dissected to separate the spleen, kidney, liver, tumour and thymus, and the weight ratio of the organ to the corresponding mice was calculated. As shown in Fig. 2, the spleen index of the saline and PEG groups was significantly increased compared with the sham group $(p<0.001)$. Compared with the PEG group, the NP1 intravenous injection group significantly reduced the spleen index $(p<0.05)$. Compared with the saline group, the NP1 intravenous injection group can significantly increase the thymus index. Compared with the saline and PEG groups, the 5 -FU group can significantly increase the thymus index $(p<0.001)$. However, the kidney index and liver index in different groups there were no statistically significant differences. Compared with the saline

B)

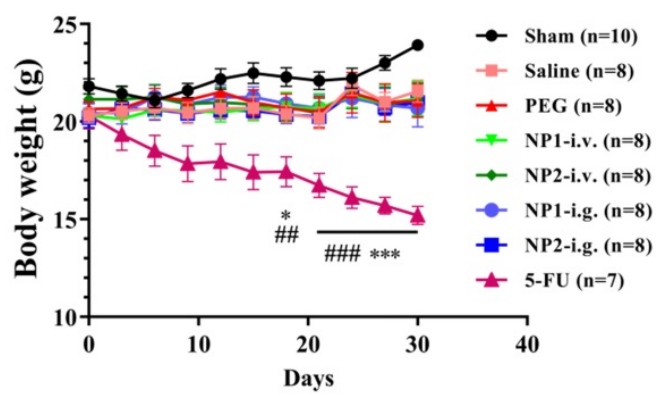

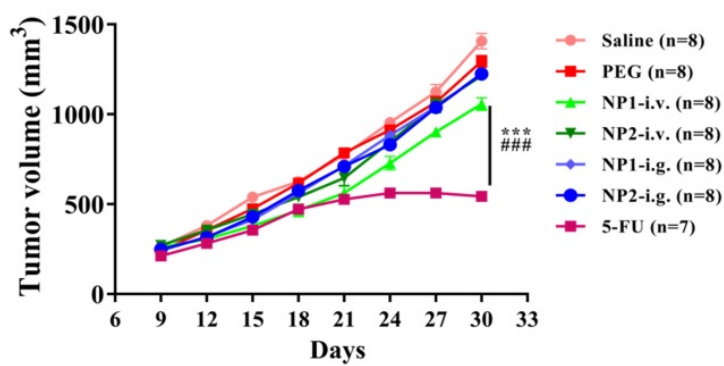

Figure 1. A) Effect of FAD on body weight change in breast mice with 4T1 cells injection. FAD was administered, b.i.d., orally or i.v. starting on 6 weeks of age continuously for a total of 4 weeks. Body weight was shown as Mean \pm SEM. ${ }^{*} p<0.05$ and $* * * p<0.001$ vs saline group mice, \# $\#<0.01$ and \#\# $\#$ m $<0.001$ vs PEG group mice. B) Effect of FAD on tumour volume in breast mice with $4 \mathrm{Tl}$ cells injection. Values were shown as Mean \pm SEM. ${ }^{* * *} \mathrm{p}<0.001$ vs saline group mice, \#\#p<0.001 vs PEG group mice. 
group, the NP1 intravenous injection group can reduce the tumour index $(\mathrm{p}<0.05)$. Compared with the saline and PEG groups, the 5-FU group can also significantly reduce the tumour index $(p<0.001)$. This behavior is probably due to specific chemical affinity of FAD conjugate to 5-FU, which induce a different steric arrangement of coenzyme and a consequent better therapeutic effect.

\section{FAD intravenous injection reduces NE, MO, $E O$, and $B A$ numbers}

Whole blood was measured with a blood cell counter before the first week of drug administration and the injection of $4 \mathrm{~T} 1$ cells (DIV 8), the second week
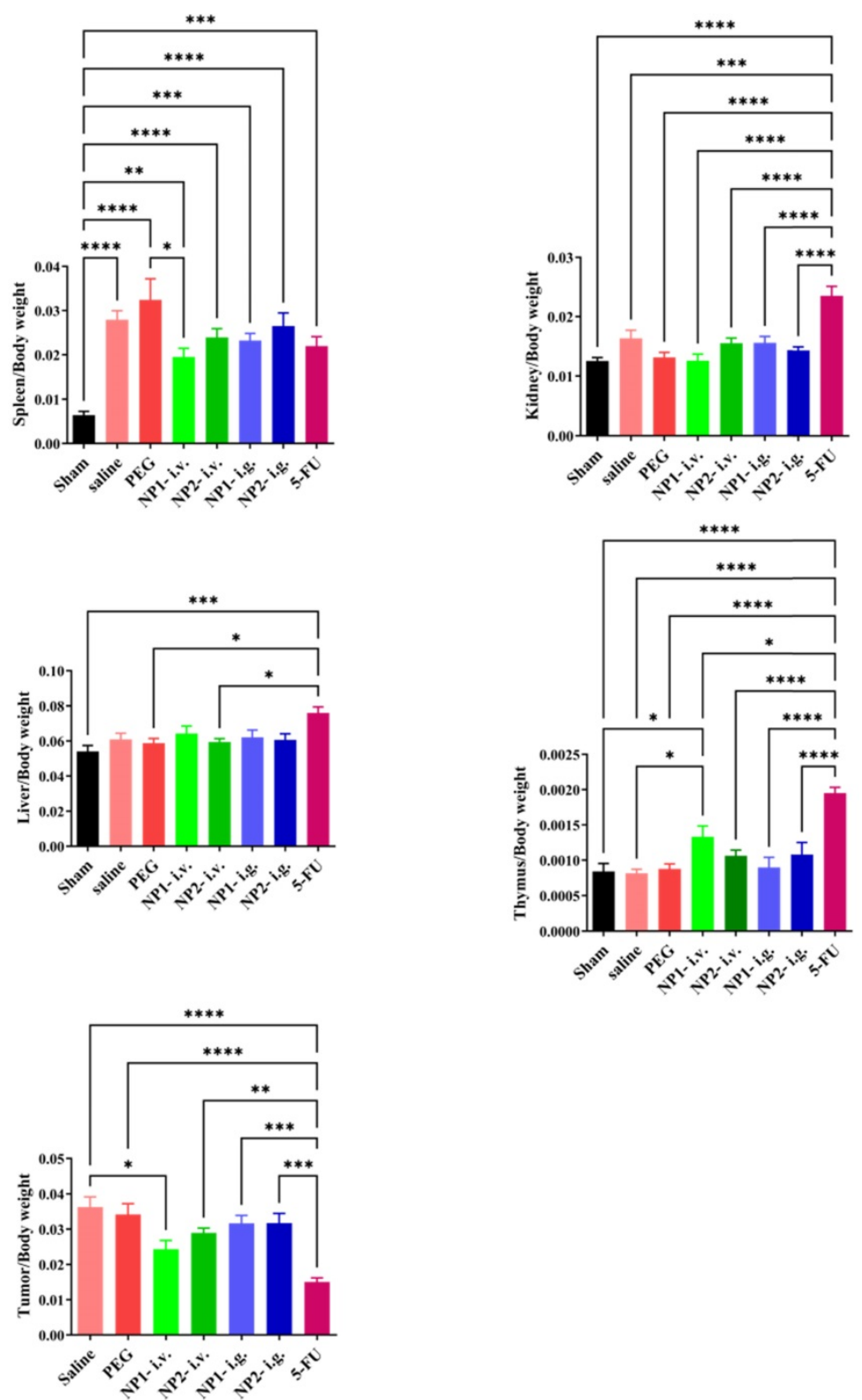

Figure 2. Effect of FAD (NPI) on Index (Spleen, Liver, Tumour, Kidney, Thymus) in breast mice with $4 T 1$ cells injection. Values were shown as Mean \pm SEM. $n=10$ for each group except sham group $(n=14)$. \&\&\&p<0.001 vs sham group mice, ${ }^{*} p<0.05$ and $* * * p<0.001$ vs saline group mice, $\# p<0.05$ and \#\# $<0.001$ vs PEG group mice. after administration (DIV 15), the third week after administration (DIV 22), and the fourth week (DIV 29). As shown in Fig. 3, the number of NE, MO, EO, and $\mathrm{BA}$ in the NP 1 intravenous group has been reduced after 4 weeks of administration. Meanwhile, all indexes except the MPV index has been reduced in the 5-FU group. Contrarily to our previous study [18] in NP1 formulation, the steric arrangement of free FAD molecules, allows a better chemical interaction with 5-FU that permit a boost enhancement of blood cells that provoke inhibition of Glucose 6 phosphate dehydrogenases (G6PD) [20].

\section{Effects of FAD (NP1) and FAD/PEG (NP2) complexes on cytokines expression}

Cytokines and chemokines are prompt proteins generated from various types of cells that regulate immune responses [22]. There are two immune responses for cytokine production: 1) Antigen presenting cells (APCs) take up antigens, process them, and consequently follow them to T-lymphocytes to cause cytokines, 2) APCs, such as monocytes, are activated to generate cytokines by pattern recognition receptors that detect an extraneous pathogen [23]. Cytokines mostly produced by APCs include multiple interleukin (IL) and tumour necrosis factor (TNF) molecules [24]. Many clinical studies have demonstrated that inoculating a standard dose of chemotherapeutic drugs causes an enhancement in cytokine levels for a kind of cytokines (TNF-a, IL-6, IL-8, IL-10 [25], and monocyte chemotactic protein-1 (MCP-1) [26]. The lack to elucidate an injury can build extreme immune cell infiltration and lead to resolute cytokine production.

Previously we have demonstrated that the combination within NP2 (FAD-PEG Diacid Complex) and 5-FU have the potential of anti-inflammatory agents as they significantly reduced the serum levels of IL-6, TNF- $\alpha$ and IL-12 P70 probably due to anti-inflammatory properties of FAD [18].

Herein, flow cytometry was used to measure cytokines levels in serum before injecting 4T1 cells (DIV 0), 1 week (DIV 8), 2 weeks (DIV 22), and 3 weeks (DIV 29). As shown in Fig. 4, the levels of IL-23, MCP-1, IL-17A and IFN $\beta$ were not different between the different groups at the corresponding time points. After 2 and 3 weeks of administration, the levels of IL-1a, GMCSF, and IL-6 were significantly increased $(p<0.01)$, but the FAD (NP1) and FAD/PEG (NP2) complexes and 
the 5-FU group did not significantly affect IL-1a, GMCSF and IL-6 levels compared with the sham group. After 3 weeks of administration, compared with the sham group, the levels of IFN $\gamma$, TNF $\alpha$, IL-12 P70, IL-1 $\beta$ and IL-27 were significantly increased, compared with the saline and PEG groups, FAD (NP1) and FAD/PEG (NP2) complexes and did not significantly improve the changes in the levels of IFN $\gamma$, TNF a, IL-1 $\beta$, and IL-27. But the FAD intravenous injection group could significantly improve the
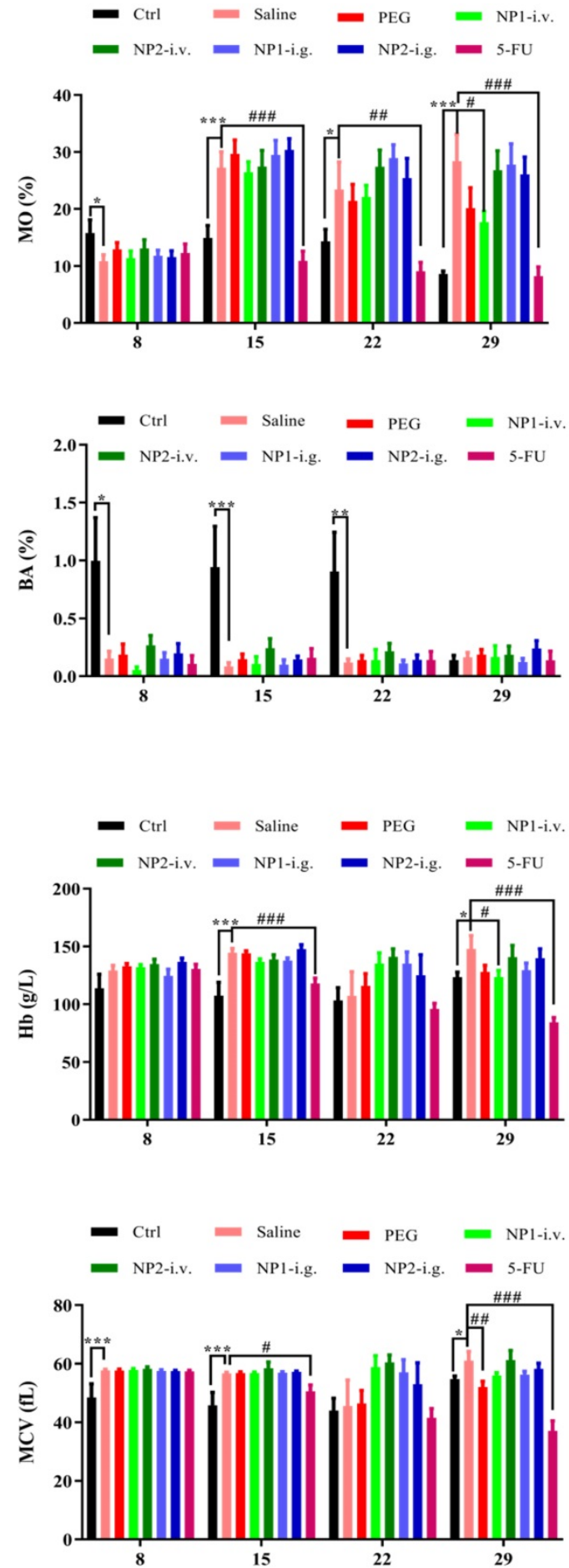

changes of IL-12 P70 ( $p<0.01)$, and 5-FU could be different significantly reduced the levels of IFN $\gamma$, TNF $\alpha$, IL-12 P70, IL-1 $\beta$, and IL-27 ( $p<0.01)$. 4 T1 cells were injected 1 week before the first drug administration (DIV 8), compared to sham group, the NP1-iv, NP 2-iv, and NP 1-ig groups significantly reduced the IL-10 levels ( $p<0.05)$ that plays a central role in regulating homeostasis resolving inflammation during acute infections or tissue injury at both a local and systemic level.
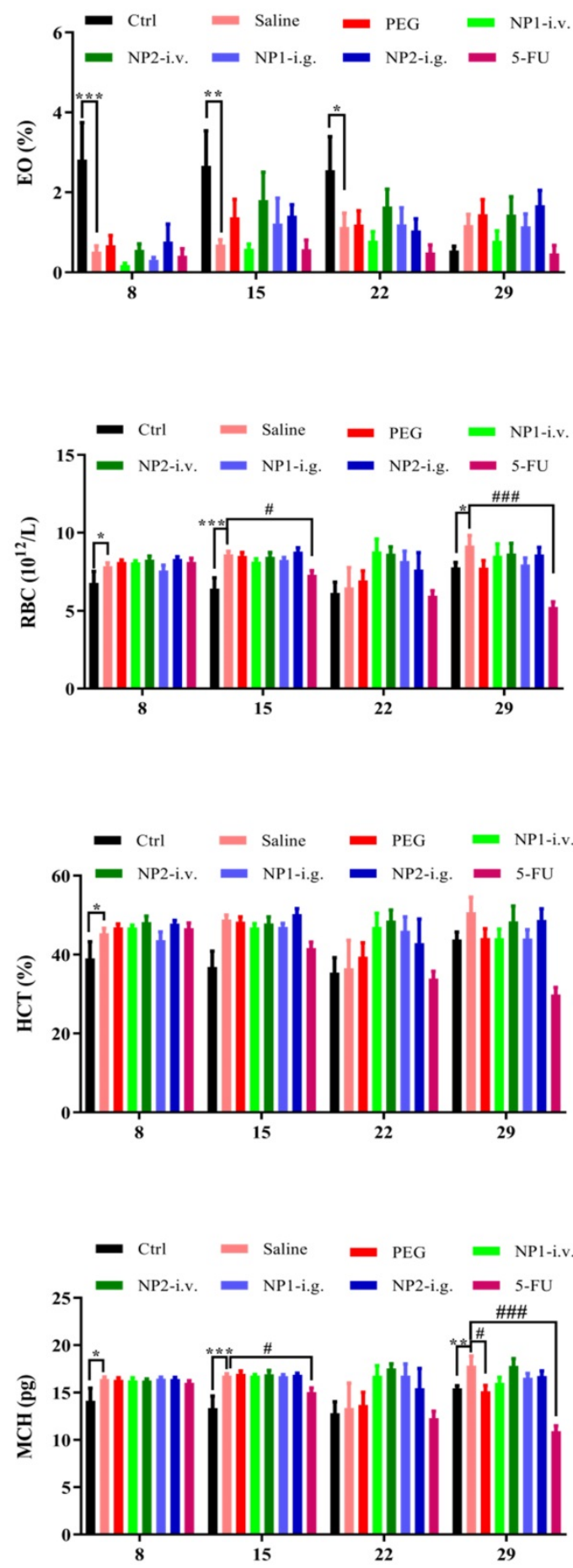

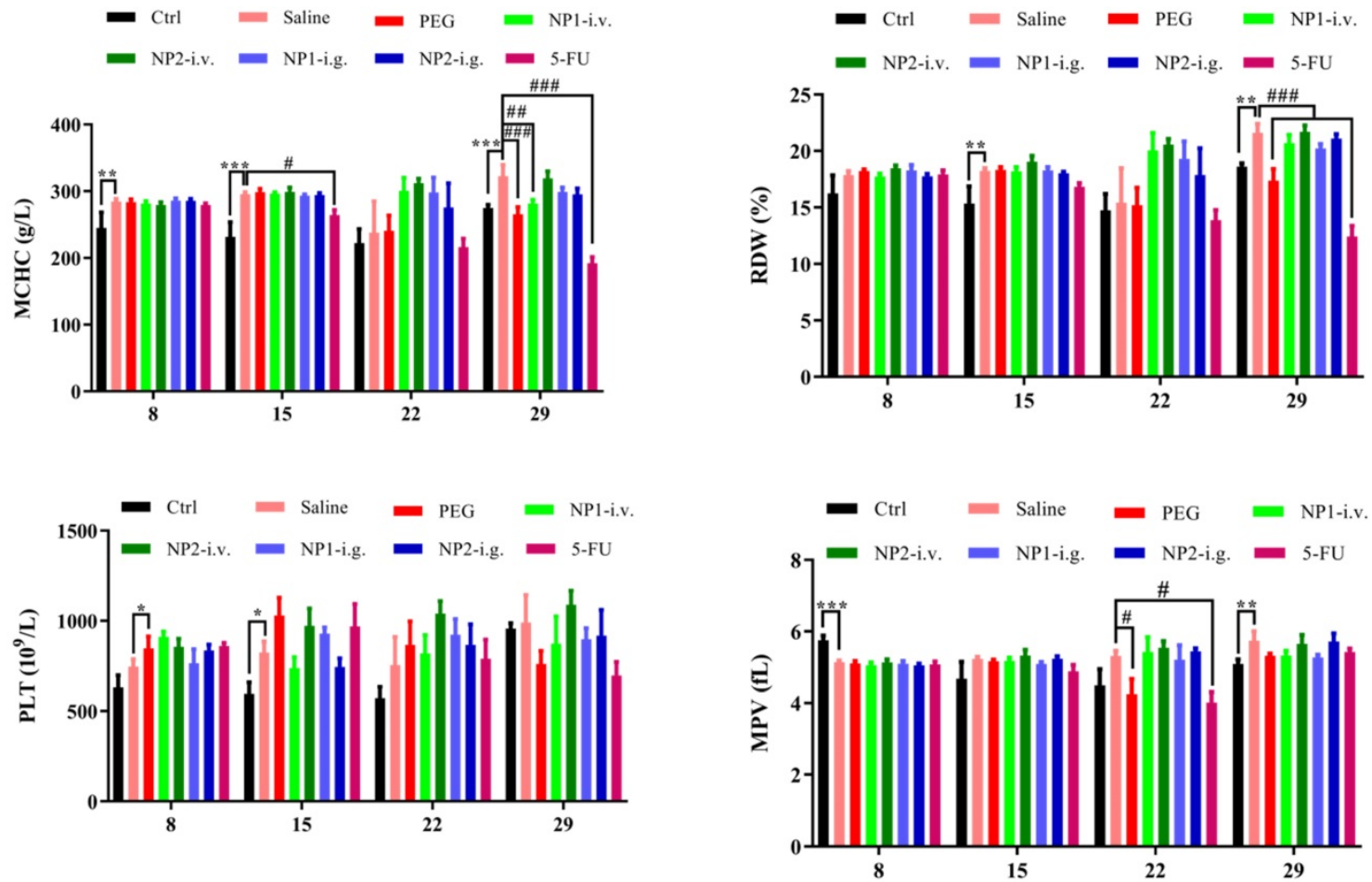

Figure 3. Effect of FAD on whole blood cell count in breast mice with $4 T 1$ cells injection.
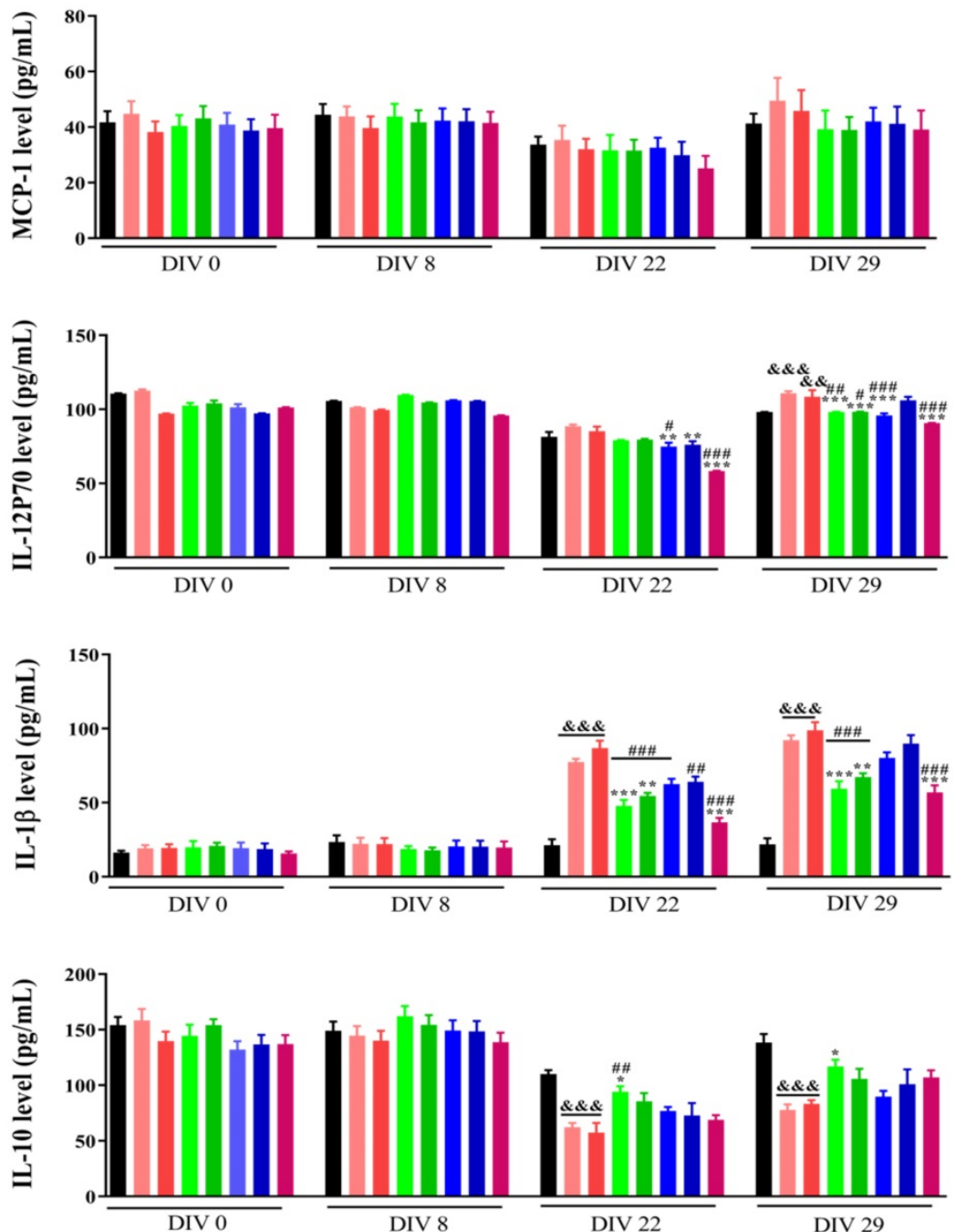

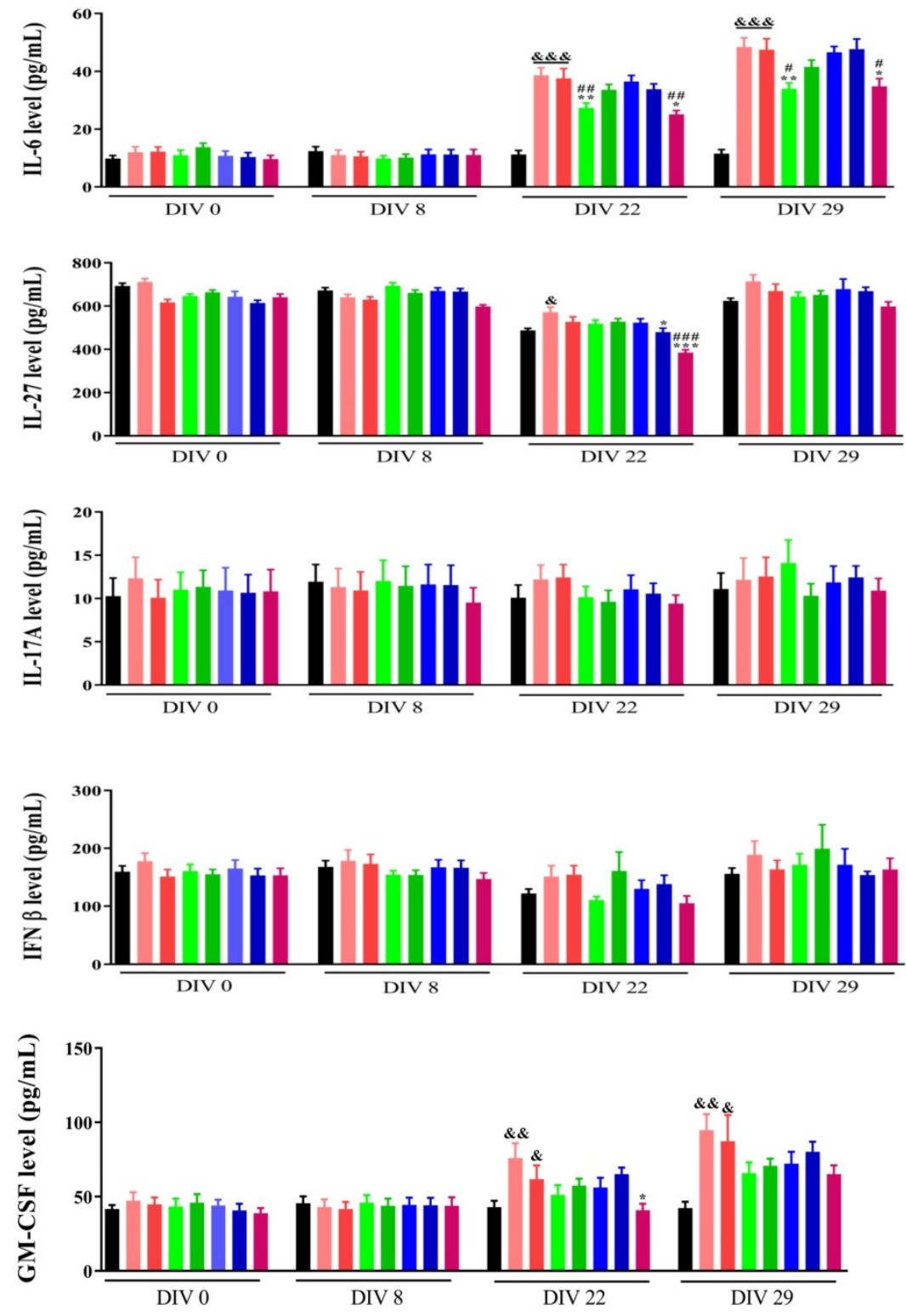

Figure 4. Effects of FAD on cytokine in breast mice with $4 \mathrm{TI}$ cells injection. Values were shown as Mean $\pm \mathrm{SEM}$. \&P $<0.05, \& \& p<0.01$ and \&\&\&p<0.001 vs sham group mice, ${ }^{*} \mathrm{p}<$ $0.05, * * p<0.01$ and $* * * p<0.001$ vs saline group mice, $\# p<0.05, \# p<0.01$ and $\# \# p<0.001$ vs PEG group mice.

On the basis of these findings, we can suggest that NP1 decrease the level of pro-inflammatory cytokines and the synergic combination between NP1 and 5-FU strongly improve immune system effects [18].

\section{Conclusions}

In this paper, we have analysed the importance of flavins coenzymes as a biocompatible formulation in a mouse model of breast cancer metabolism, as an adjuvant, and as an anticancer drug. Contrary to our previous paper [18] of the effect of FAD (NP1) and FAD/PEG (NP2), where the complexes have a booster effect on a murine model of liver cancer, on our murine model of breast cancer only intravenous injection of FAD can significantly reduce tumour volume, tumour index and increase thymus index; reduce neutrophils (NE), monocytes (MO), eosinophils (EO), and basophils (BA). At the same time, the content of IL-1 $\alpha$, IL-12 P70, TNF $\alpha$, IL-1 $\beta$ and IL-6 was significantly reduced, and the content of IL-10 was significantly increased like in the previous liver cancer model. The advanced antitumor efficacy of FAD (NP1) compared to 5-FU is displayed, not only in the repression of tumour growth, but similarly in the stimulation of immune system. The signal pathway of FAD acting on blood cells/immune cells/tumour cells and the analysis of tumour tissue or immune cells will be studied in genomics and proteomics. These results predict the wave for the development of an innovative theranostic platform, allowing the detection of protein-associated tumours 
and the simultaneous cancer treatment with an adjuvant that reduce secondary effect of chemotherapy and boost immunity system with a consequent good replication of healthy cells.

\section{Supplementary Material}

Supplementary figure and table. http://www.ntno.org/v06p0175s1.pdf

\section{Competing Interests}

The authors have declared that no competing interest exists.

\section{References}

1. Siegel RL, et al. Cancer statistics, 2020. CA: A Cancer Journal for Clinicians. 2020;70 (1): 7-30

2. Fitzmaurice $\mathrm{C}$, et al. Global, Regional, and National Cancer Incidence, Mortality, Years of Life Lost, Years Lived with Disability, and Disability-Adjusted Life-Years for 29 Cancer Groups, 1990 to 2016: A Systematic Analysis for the Global Burden of Disease Study. JAMA oncology. 2018;4(11):1553-1568

3. Falzone LS. Evolution of Cancer Pharmacological Treatments at the Turn of the Third Millennium.Frontiers in pharmacology. 2018; 9: 1300.

4. Moustaoui HM-M, et al. Tunable Design of Gold(III)-Doxorubicin Complex-PEGylated Nanocarrier. The Golden Doxorubicin for Oncological Applications. ACS applied materials \& interfaces. 2016;8(31):19946-19957.

5. Ruman U, et al. Nanocarrier-Based Therapeutics and Theranostics Drug Delivery Systems for Next Generation of Liver Cancer Nanodrug Modalities. Int J Nanomedicine. 2020;15:1437-1456.

6. Molano-Arevalo JC. Flavin Adenine Dinucleotide Structural Motifs: From Solution to Gas Phase. Analytical Chemistry. 2014;86 (20):10223-10230.

7. Mansoorabadi SO, et al. The diverse roles of flavin coenzymes--nature's most versatile thespians. J Org Chem. 2007;72 (17): 6329-6342

8. Ramsay RR. Electron carriers and energy conservation in mitochondrial respiration. ChemTexts. 2019;5 (2):9.

9. Giancaspero TA, et al. Human FAD synthase is a bi-functional enzyme with a FAD hydrolase activity in the molybdopterin binding domain. Biochem Biophys Res Commun. 2015;465 (3):443-9.

10. Leone $\mathrm{P}$, et al. The hidden side of the human FAD synthase 2. Int J Biol Macromol. 2019:138: 986-995.

11. Brea-Calvo $\mathrm{G}$, et al. Chemotherapy induces an increase in coenzyme Q10 levels in cancer cell lines. Free Radic Biol Med. 2006;40 (8):1293-302.

12. Giancaspero TA, et al. The occurrence of riboflavin kinase and FAD synthetase ensures FAD synthesis in tobacco mitochondria and maintenance of cellular redox status. The FEBS Journal. 2009;276 (1):219-231.

13. Giancaspero TA, et al. FAD synthesis and degradation in the nucleus create a local flavin cofactor pool. The Journal of Biological Chemistry. 2013;288(40):2906

14. Oermann EK, et al. Alterations of metabolic genes and metabolites in cancer. Semin Cell Dev Biol. 2012;23 (4):370-380.

15. Chaturvedi $S$, et al. Human Metabolic Enzymes Deficiency: A Genetic Mutation Based Approach. Scientifica (Cairo). 2016;p:9828672-9828672.

16. Sinkala $\mathrm{M}$, et al. Metabolic gene alterations impact the clinical aggressiveness and drug responses of 32 human cancers. Communications Biology. 2019;2 (1):414.

17. Didier Paleni and Jolanda Spadavecchia . Flavin adenine dinucleotide (fad) for use in the prevention and/or treatment of cancer. 2020; PCT/EP2019/079693, WO/2020/089310.

18. Arib C, Liu H, Liu Q, Cieutat AM, Paleni D, Li X and Spadavecchia JA. Pegylated Flavin Adenine Dinucleotide PEG Complex to Boost Immunogenic and Therapeutic Effects in liver Cancer Model. Nanotheranostics. 2021; 5(4): 405-416.

19. Jia L, Liu X. The conduct of drug metabolism studies considered good practice (II): in vitro experiments. Curr Drug Metab. 2007;8 (8):822-829.

20. Luzzatto L, Nannelli C, Notaro R. Glucose-6-Phosphate Dehydrogenase Deficiency. Hematol Oncol Clin North Am. 2016;30 (2):373-93

21. Yoshida $\mathrm{H}$, et al. X-ray structure of the direct electron transfer-type FAD glucose dehydrogenase catalytic subunit complexed with a hitchhiker protein. Acta Crystallographica Section D. 2019;75 (9):841-851

22. Ramesh G, et al. Cytokines and chemokines at the crossroads of neuroinflammation, neurodegeneration, and neuropathic pain. Mediators Inflamm.2013; p:480739-480739.

23. Gaudino SJ, Kumar P. Cross-Talk Between Antigen Presenting Cells and T Cells Impacts Intestinal Homeostasis, Bacterial Infections, and Tumorigenesis. Front Immunol. 2019:10:360-360.
24. Turner $\mathrm{MD}$, et al. Cytokines and chemokines: At the crossroads of cell signalling and inflammatory disease. Biochimica et Biophysica Acta (BBA) Molecular Cell Research. 2014;1843 (11):2563-2582.

25. Tanaka T, Narazaki M, Kishimoto T. IL-6 in inflammation, immunity, and disease. Cold Spring Harb Perspect Biol. 2014;6(10), a016295-a016295.

26. Groves TR, et al. 5-Fluorouracil chemotherapy upregulates cytokines and alters hippocampal dendritic complexity in aged mice. Behav Brain Res. 2017:316:215-224. 